\title{
Melting Curve and Isostructural Solid Transition in Superionic Ice
}

\author{
J.-A. Queyroux, ${ }^{1,2}$ J.-A. Hernandez $\odot,{ }^{3}$ G. Weck,${ }^{2}$ S. Ninet, ${ }^{1}$ T. Plisson $\odot,{ }^{2}$ S. Klotz, ${ }^{1}$ \\ G. Garbarino, ${ }^{4}$ N. Guignot, ${ }^{5}$ M. Mezouar, ${ }^{4}$ M. Hanfland, ${ }^{4}$ J.-P. Itié $\odot,{ }^{5}$ and F. Datchi $\odot^{1, *}$ \\ ${ }^{1}$ Institut de Minéralogie, de Physique des Matériaux et de Cosmochimie (IMPMC), Sorbonne Université, \\ CNRS UMR 7590, Muséum National d'Histoire Naturelle, 4 place Jussieu, F-75005 Paris, France \\ ${ }^{2}$ Commissariat à l'Energie Atomique (CEA), Direction des Applications Militaires (DAM), DAM Ile-de-France (DIF), \\ F-91297 Arpajon, France \\ ${ }^{3}$ Centre for Earth Evolution and Dynamics, University of Oslo, 1028 Blindern, N-0315 Oslo, Norway \\ ${ }^{4}$ European Synchrotron Radiation Facility, BP 220, F-38043 Grenoble Cedex, France \\ ${ }^{5}$ Synchrotron Soleil, L'Orme des Merisiers, Saint-Aubin, BP 48, 91192 Gif-sur-Yvette Cedex, France
}

(Received 22 July 2020; accepted 28 September 2020; published 2 November 2020)

\begin{abstract}
The phase diagram and melting curve of water ice is investigated up to $45 \mathrm{GPa}$ and $1600 \mathrm{~K}$ by synchrotron $\mathrm{x}$-ray diffraction in the resistively and laser heated diamond anvil cell. Our melting data evidence a triple point at $14.6 \mathrm{GPa}, 850 \mathrm{~K}$. The latter is shown to be related to a first-order solid transition from the dynamically disordered form of ice VII, denoted ice VII', toward a high-temperature phase with the same bcc oxygen lattice but larger volume and higher entropy. Our experiments are compared to ab initio molecular dynamics simulations, enabling us to identify the high-temperature bcc phase with the predicted superionic ice VII" phase [J.-A. Hernandez and R. Caracas, Phys. Rev. Lett. 117, 135503 (2016).].
\end{abstract}

DOI: 10.1103/PhysRevLett.125.195501

Ice is well known for its remarkable structural diversity in the low pressure $(P \leq 2 \mathrm{GPa})$, low temperature $(T \leq 270 \mathrm{~K})$ range. Above $2 \mathrm{GPa}$, however, all known phases of ices are constructed on the same body-centered cubic (bcc) lattice of oxygen atoms which remains stable up to extreme pressures-at least $150 \mathrm{GPa}$ at room temperature [1], and up to the melting line. The main structural differences between these bcc-based ices thus only reside in the average position and the dynamics of the $\mathrm{H}$ atoms, as described below.

In the low temperature ice VIII, the water dipoles, and thus $\mathrm{H}$ atoms, are fully ordered, inducing a slight tetragonal distortion of the bcc lattice [2]. This order disappears at the transition to ice VII ( $T=273 \mathrm{~K}$ at $2-8 \mathrm{GPa}$, decreasing to $0 \mathrm{~K}$ at $\sim 60 \mathrm{GPa}$ ), which may be characterized as an orientationally disordered crystal: the $\mathrm{H}$ atoms may occupy any of the half-diagonals of the bcc unit cell, while respecting the Bernal-Fowler (ice) rules [2,3]. Above $40 \pm 10 \mathrm{GPa}$ at $300 \mathrm{~K}$, the probability for translational movements of $\mathrm{H}$ atoms along the $\mathrm{O}-\mathrm{H} \cdots \mathrm{O}$ bonds becomes substantial, leading to a bimodal distribution of the protons along the bcc half-diagonal. This regime has been denoted dynamic translationally disordered ice VII, or ice VII' [4-6]. At $\sim 80 \mathrm{GPa}$, the proton distribution becomes unimodal again and peaks midway along the half-diagonal: this is the symmetric ice $\mathrm{X}$ [7]. It is worth noting that the VII-VII'-X transition sequence occurs without any discontinuous change of density $[1,8,9]$.

With increasing temperature, the mobility of $\mathrm{H}$ atoms is enhanced, which lowers the transition pressure from VII to
VII'. Close to the melting line, another type of dynamical events is favored according to recent theoretical studies [6,10-12]: $\mathrm{H}_{2} \mathrm{O}$ rotations. In the moderate $P-T$ range $(P<15 \mathrm{GPa}, T<800 \mathrm{~K})$, this gives rise to a plastic form of bcc ice, composed of free $\mathrm{H}_{2} \mathrm{O}$ rotors (yet to be confirmed by experiments). At higher $P-T, \mathrm{H}$ translations and rotations coexist, and $\mathrm{H}$ diffusion across the $\mathrm{O}$ lattice become possible. This is the superionic (SI) state, initially observed in $a b$ initio molecular dynamics (AIMD) simulations above $30 \mathrm{GPa}, 2000 \mathrm{~K}[13,14]$. The more recent AIMD work [10] predicts that superionicity should appear at the milder $P-T$ conditions of $15 \mathrm{GPa}-1000 \mathrm{~K}$, in a form of bcc ice denoted VII". SI-Ice VII" is stable along the melting line above $1000 \mathrm{~K}$ and differs from ice VII' by a stronger delocalization of protons. Interestingly, Hernandez and Caracas [10] found that SI-ice VII" transits to ice VII on compression, and this isostructural transition is first order. Close to the transition pressure, ice VII' is also superionic, but the electronic conduction is smaller than in ice VII" and gradually vanishes, in a continuous fashion, under further compression.

Experimental evidence for SI ice has been claimed by several authors, either from static $[4,15]$ or laser shock experiments $[16,17]$. Yet the specific conditions at which bcc ice becomes superionic remain strongly controversial. Since the early prediction of Ref. [13], it has been recognized that the important entropy increase in the SI state should translate in a significant change of slope of the melting curve at the liquid-ice-SI-ice triple point. Such a kink was indeed reported in some experiments around 
$40 \mathrm{GPa}[4,18,19]$. However, there are at present large uncertainties on the melting curve of ice due to inconsistent experimental results above $15 \mathrm{GPa}[4,18-26]$ : a difference of $300 \mathrm{~K}$ exists between the lowest and the highest reported melting temperature at $20 \mathrm{GPa}$, increasing to $800 \mathrm{~K}$ at $40 \mathrm{GPa}$.

In this Letter, we report new measurements of the melting curve and phase diagram of ice from $8.4 \mathrm{GPa}-$ $660 \mathrm{~K}$ to $45 \mathrm{GPa}-1450 \mathrm{~K}$, based on synchrotron $\mathrm{x}$-ray diffraction (XRD). These provide strong evidence for a first-order transition between two bcc forms of ice, associated to a change of slope of the melting curve at 14.6 GPa, $850 \mathrm{~K}$, and an important increase of entropy. We find excellent agreement between the presently observed high-temperature bcc phase with the predicted SI-ice VII", and thus identify the kink on the melting line as the liquid-VII' - VII" triple point.

Present experiments were performed on water samples compressed in diamond anvil cells. The complete experimental methods are given in the Supplemental Material file (SM) [27]. For temperatures up to $1000 \mathrm{~K}$, we used resistive heating $(\mathrm{RH})$ which enables a homogeneous heating of the sample and accurate $P-T$ measurements. Above $1000 \mathrm{~K}$, samples were laser heated $(\mathrm{LH})$ by the $10.6 \mu \mathrm{m}$ radiation of a $\mathrm{CO}_{2}$ laser which is directly absorbed by water. Water samples were only in contact with the diamond anvils and gold during the experiments, and no evidence for chemical reaction with these elements was observed in the investigated $P-T$ range. Temperature in $\mathrm{LH}$ runs was determined by spectrally analyzing the sample thermal emission. The absence of insulation layers between water and diamond anvils implied the presence of a large thermal gradient along the diamond anvil cell axis. Simulations of various temperature profiles indicate that the Planck temperature reported here is, within 5\%, that of the hottest part of the sample (see Sec. I. B and Fig. S1 of the SM [27]). Note that a radial thermal gradient also exists across the hot spot but is less problematic thanks to the small x-ray beam size $(3 \times 3 \mu \mathrm{m}$ FWHM at ID27) compared to the hot spot diameter $(\sim \varnothing 40 \mu \mathrm{m})$. Angular dispersive X-ray diffraction (XRD) experiments were performed at the ID27 beam line of the European Synchrotron Radiation Facility (ESRF, Grenoble, France) for LH runs, and at the PSICHE beam line of the SOLEIL synchrotron (Saint Aubin, France) for the RH runs. Some melting data were also obtained in RH experiments on the ID09 (now ID15B) beam line of ESRF. The x-ray wavelength was $0.3738 \AA$ in all cases.

Melting curve of ice.-Melting of ice was investigated from 8.4 to $44 \mathrm{GPa}$. The results are displayed and compared to literature in Fig. 1 (see also Table S1 of the SM [27]). RH runs consisted in either isothermal or quasi-isobaric paths. Melting was inferred by the disappearance of the Bragg diffraction peaks from ice and the simultaneous appearance of the broad diffuse scattering ring of water on the XRD

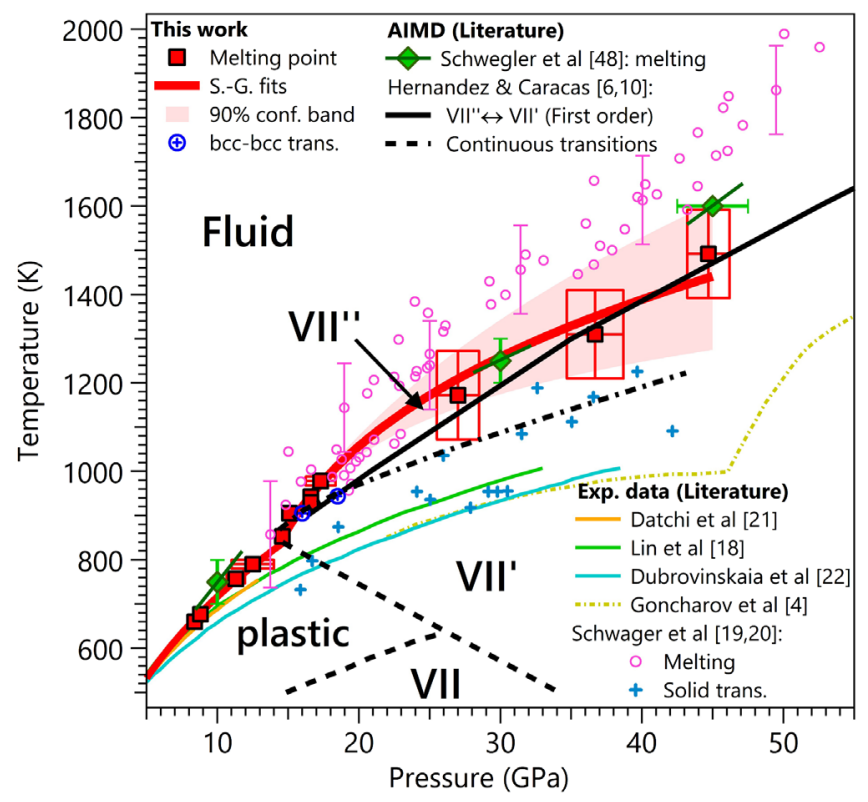

FIG. 1. Phase diagram of $\mathrm{H}_{2} \mathrm{O}$. Red squares and blue circles: experimental data for the melting line and the isostructural solid transition, respectively; red line: Simon-Glatzel fit to the melting data; pale red zone: $90 \%$ confidence band of the fit. Thick solid and dashed lines: AIMD transition lines from Ref. [6]. Dashdotted line: guide to the eye for the solid transition line based on this work and Ref. [20]. Experimental [4,19-22,24] and AIMD data [48] from literature are also represented.

images. As shown in Fig. 2(a), the liquid diffuse ring appears clearly after subtraction of a XRD pattern collected below the melting temperature, as this removes the background (Compton) contribution. At a given temperature, the melting pressure was defined as the midpoint of the pressure interval where melting was observed.

LH experiments exclusively consisted of quasi-isobaric heating runs where laser power is ramped up or down at fixed load. The detection of melting was similar as above, although in this case the diffraction peaks from the solid phase do not completely disappear at melting due to the axial thermal gradient discussed above. Figures 2(b) and 2(c) show the broad diffuse scattering which appears around the (110) peak of the bcc ice at melting, whose intensity increases as the temperature ramps up due to a larger amount of melt in the hot spot.

As seen in Fig. 1, up to $\sim 13 \mathrm{GPa}$ our results are in best agreement with the RH experiments of Refs. [21,24], the differences being likely due to the different means of measuring pressure. Above $\sim 13 \mathrm{GPa}$, our melting points from both our RH and LH experiments increasingly deviate from all previous $\mathrm{RH}$ experiments and better match the $\mathrm{LH}$ experiments of Refs. [19,20]; the agreement is within mutual uncertainties, although our melting temperatures are systematically $100-150 \mathrm{~K}$ lower. Our data are also in excellent agreement with the theoretical results of Schwegler et al. [48], who used the two-phase simulation 


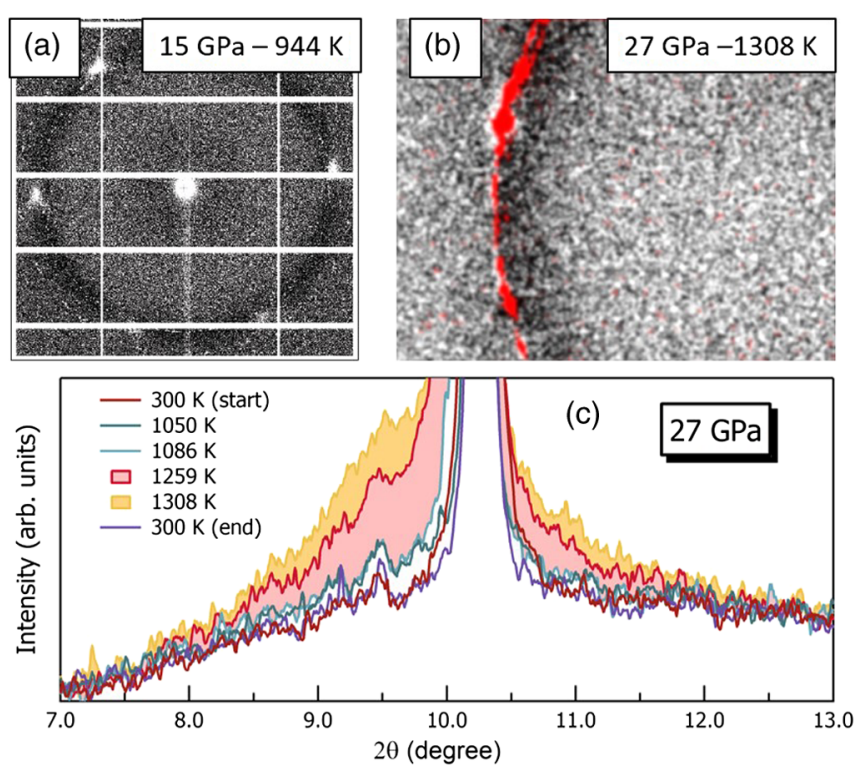

FIG. 2. Melting of ice seen by XRD. (a) and (b): XRD images above the melting temperature after subtraction of a room temperature image, at (a) $15.4 \mathrm{GPa}-944 \mathrm{~K}$ in a $\mathrm{RH}$ run and (b) $27 \mathrm{GPa}-1308 \mathrm{~K}$ in a $\mathrm{LH}$ run. The white stripes in (a) are inactive parts of the detector. The red pixels in (b) correspond to diffraction from ice. (c) Integrated patterns at $27 \mathrm{GPa}$ and several temperatures. The liquid signal appears as a broad peak around $10^{\circ}$ at $1260 \mathrm{~K}$.

method coupled to AIMD. The reasons why present data are so discrepant from previous RH experiments above $13 \mathrm{GPa}$ are not obvious; in particular, Refs. [22] and [23] also used a XRD criteria for melting, and yet report melting temperatures lower than ours by several hundred K. We note that these authors did not report the observation of the liquid diffuse ring and thus solely associated melting with the disappearance of the Bragg peaks of ice; this may be misleading as the disappearance of Bragg peaks may also be due to the recrystallization of the ice sample into a few single crystals below the melting point.

The present data highlight a discontinuous change of slope of the melting curve around $14.6 \mathrm{GPa}$ and $850 \mathrm{~K}$. The data were thus fit separately below and above 14.6 GPa, using the Simon-Glatzel equation [49] (see Fig. 1 and Table I). The observed change of slope indicates the presence of a triple point at 14.6(5) $\mathrm{GPa}$ and $850(20) \mathrm{K}$. This is consistent with our observations of a

TABLE I. Fit parameters of the Simon-Glatzel equation $T_{m}\left(P_{m}\right)=T_{t}\left[\left(P_{m}-P_{t}\right) / a+1\right]^{(1 / b)}$. Numbers in parentheses give the $90 \%$ confidence interval on the last digit.

\begin{tabular}{lcccl}
\hline \hline & $a$ & $b$ & $P_{t}(\mathrm{GPa})$ & $T_{t}(\mathrm{~K})$ \\
\hline$P \leq 14.6 \mathrm{GPa}$ & $1.555(14)$ & $2.557(14)$ & $2.17^{\mathrm{a}}$ & $354.8^{\mathrm{a}}$ \\
$P \geq 14.6 \mathrm{GPa}$ & $3.44(216)$ & $4.33(200)$ & 14.6 & 850 \\
\hline \hline
\end{tabular}

${ }^{\mathrm{a} V I-V I I-f l u i d ~ t r i p l e ~ p o i n t ~ c o o r d i n a t e s ~ f r o m ~ R e f . ~[21] . ~}$
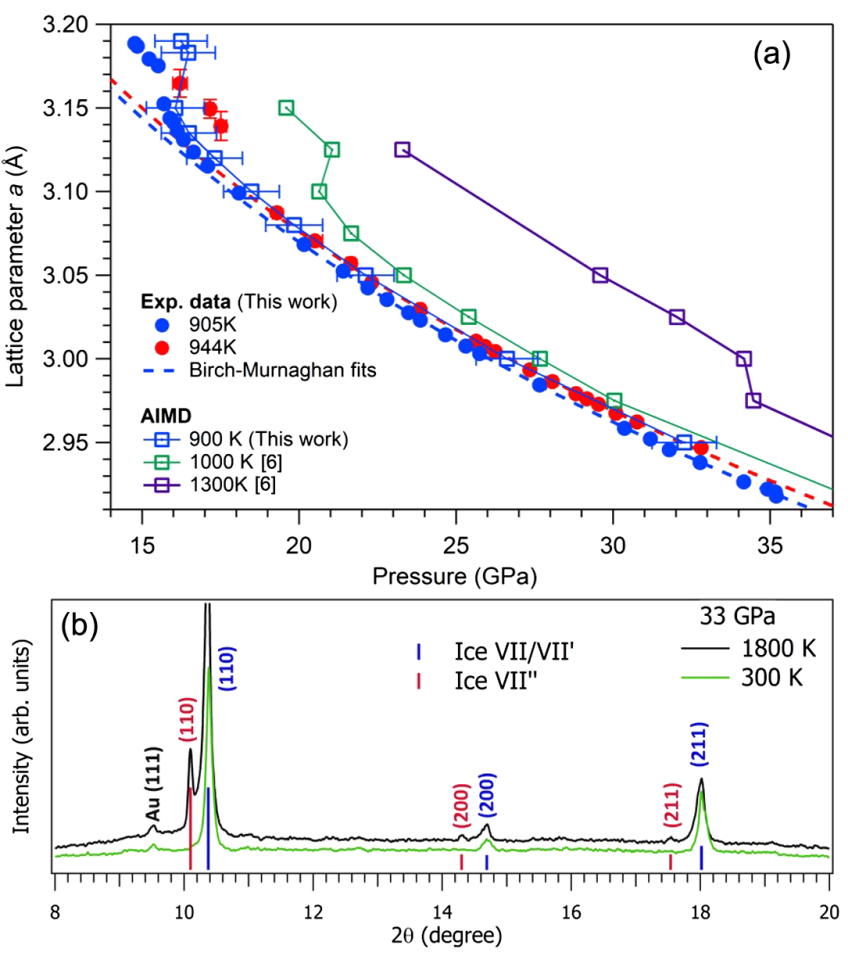

FIG. 3. Isostructural transition. (a) Lattice parameter of ice as a function of pressure above $900 \mathrm{~K}$, showing the discontinuous jump at the $\mathrm{VII}^{\prime \prime}-\mathrm{VII}^{\prime}$ transition. Dots: experimental data; squares: AIMD data. Dashed lines: Birch-Murnaghan equation of state fits to experiment for $P \gtrsim 20 \mathrm{GPa}$. (b) XRD patterns of ice at $33 \mathrm{GPa}$ from a $\mathrm{LH}$ run. The second set of bcc peaks present on the $1800 \mathrm{~K}$ pattern is assigned to ice VII".

first-order transition between two bcc forms of ice, as presented below.

Isostructural transition.-XRD patterns of ice were collected in a RH run along two isotherms at 905 and $944 \mathrm{~K}$, from the melting pressures up to 39 and $33 \mathrm{GPa}$, respectively. At all $P-T$ conditions, the pattern is consistent with the $P n-3 m$ structure of ice VII but, as seen in Fig. 3(a), a discontinuous shift of the lattice parameter $a$ of about $-0.7(1) \%$ - corresponding to a volume shift of $-2.2(1) \%$-is observed on both isotherms, at 15.6(2) GPa at $905 \mathrm{~K}$, and 18.4(9) GPa at $944 \mathrm{~K}$ (see also Fig. S2 of the $\mathrm{SM})$. At higher pressures, $a$ varies continuously with $P$ and can be fitted by a single Birch-Murnaghan equation of state [50] [see Fig. 3(a) and Table S2 in the SM [27]]. This strongly suggests the occurrence of a phase transition between ice VII' and another bcc form of ice before melting. The latter was also evidenced in our LH runs. Figure 3(b) shows an example of diffraction pattern collected at $33 \mathrm{GPa}$, at 300 and $1800 \mathrm{~K}$; in addition to the diffraction peaks of ice VII, smaller ones are present at $1800 \mathrm{~K}$ on the low $2 \theta$ side of (110), (200), and (311). These peaks (or a subset of them) were observed at all investigated pressures from 27 to $44 \mathrm{GPa}$ and reproducibly appeared and disappeared when temperature was cycled. 
They thus cannot originate from a chemical reaction or a dissociation of water; this was confirmed by Raman measurements on a sample at $34 \mathrm{GPa}-300 \mathrm{~K}$ after a $\mathrm{LH}$ run, which only displayed the Raman bands from ice VII (see Fig. S3 of the SM [27]). The additional Bragg peaks can be indexed by a bcc unit cell, confirming the presence of a second bcc solid with a larger volume than ice VII. We estimate a volume difference of $2.5 \%$ at $33 \mathrm{GPa}$ (see Fig. S4 of the SM [27]), in good agreement with our RH data. The peaks appeared at temperatures where the liquid signal started to be seen, showing that the solid phase transition occurs at a temperature close to the melting line. The actual transition temperature is difficult to determine due to the axial thermal gradient and because the measured temperature corresponds to that of the hottest part of the sample. Moreover, it was difficult to perform small temperature steps, i.e., below $\sim 100 \mathrm{~K}$, in $\mathrm{LH}$ experiments.

The solid transition points obtained in our RH experiments, plotted in Fig. 1, coincide within uncertainties with a transition previously reported by Schwager and Boehler [20]. These authors deduced the occurrence of a solid transition solely from visual observations, but no information on the nature of this high-temperature (HT) phase has been made available so far. We show here that the latter keeps the oxygen bcc lattice of ice VII and has a larger volume. Therefore, the structure of the HT phase must only differ from that of ice VII in the positions of the $\mathrm{H}$ atoms. Moreover, we determine from the Clapeyron relation an entropy increase $\Delta S$ of 1.7(6) $R$ across the transition, where $R$ is the ideal gas constant. This implies a larger number of possible proton positions, and thus higher proton disorder in the HT phase. For comparison, the present value of $\Delta S$ is intermediate between those measured in ammonia [51] at the ordered-plastic solid transition $[1.0(4) R]$ and the ordered-superionic solid transition [4(2)R].

The above observations bear strong resemblance with the AIMD simulations of bcc ice in Ref. [10]. Indeed, the ice VII" phase observed in the latter work matches very well the HT phase revealed by our experiments: it is isostructural to ice VII, is stable along the melting line, and is more disordered than ice $\mathrm{VII}^{\prime}$ to which it transits at higher pressures through a first-order process. Ice VII" was only studied above $1000 \mathrm{~K}$ in Refs. [6,10], so we performed new simulations at $900 \mathrm{~K}$ using the same methods (see SM [27]). As seen in Fig. 3(a), the calculated evolution with pressure of the lattice parameter $a$ at $900 \mathrm{~K}$ shows excellent agreement with experiment. A discontinuous jump of $a$ of $-0.9(1) \%$ is predicted at $\sim 16 \mathrm{GPa}$, very close to the experimental one $[-0.7(1) \%]$. The calculated isotherms at 1000 and $1300 \mathrm{~K}$ [6] display a similar discontinuity at 21 and $34 \mathrm{GPa}$, respectively. We also note that the simulated ice with $a=3.19 \AA$ at $900 \mathrm{~K}$ eventually melted (after $15 \mathrm{ps}$ of simulation), in excellent accord with our experimental melting point at $905 \mathrm{~K}$. Ice $\mathrm{VII}^{\prime \prime}$ is not observed in simulations at $800 \mathrm{~K}$, placing the triple point between

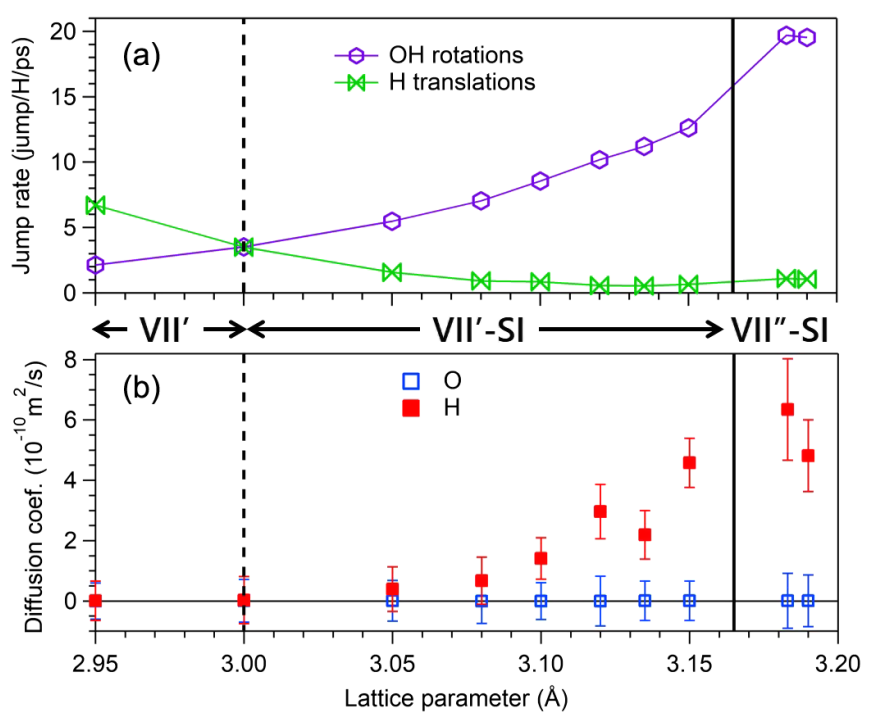

FIG. 4. $\mathrm{H}$ atom dynamics in bec ice at $900 \mathrm{~K}$ as a function of pressure from AIMD simulations. (a) $\mathrm{OH}$ rotation and $\mathrm{H}$ translation rates; (b) $\mathrm{H}$ and $\mathrm{O}$ atoms diffusion coefficients.

the liquid, ice VII' and VII" between 800 and $900 \mathrm{~K}$, in line with the kink in our melting data at $850 \mathrm{~K}$.

As discussed in Refs. $[6,10]$, ice VII' and VII" differ in the dynamics and average position of the $\mathrm{H}$ atoms. In VII', protons remain on average localized along the diagonals of the bcc oxygen sublattice, whereas in $\mathrm{VII}^{\prime \prime}$ they strongly delocalize out of the diagonals. Ice VII" is always superionic, with the highest electrical conductivity [estimated at 34(5) $\mathrm{S} \mathrm{cm}^{-1}$ at $900 \mathrm{~K}, 16.5 \mathrm{GPa}$, see Fig. S5 of the SM [27] ], while ice VII' is only superionic close to the $\mathrm{VII}^{\prime}-\mathrm{VII}^{\prime \prime}$ transition. Figure 4 illustrates the evolution of the proton dynamics and diffusion coefficient $\left(D_{H}\right)$ obtained by AIMD. $D_{H}$ starts to increase significantly for $a>3 \AA$, when the $\mathrm{OH}$ rotation rate itself increases and outruns translations. This is the VII'-SI regime. The transition to $\mathrm{VII}^{\prime \prime}$ is principally marked by a sudden increase in $\mathrm{OH}$ rotations at $a \simeq 3.165 \AA$, and a change to a constant behavior of $D_{H}$ vs $a$. These observations are fully consistent with those made at higher $T$ in Ref. [6]. Unfortunately, information on the $\mathrm{H}$ positions cannot be deduced from present XRD experiments as the contribution from $\mathrm{H}$ atoms to the diffracted signal is very weak. We note though that the increase of $D_{H}$ for $a>3 \AA$ correlates well with the progressive deviation of the experimental $a(P)$ data at $905 \mathrm{~K}$ from the Birch-Murnaghan equation of state below $20 \mathrm{GPa}$, as seen in Fig. 3(a). This can be understood by the increasing delocalization of the protons along the body diagonals when entering the VII'-SI regime, inducing an anomalous expansion of the lattice. The further, discontinuous expansion at the transition to ice VII" is explained by the sudden delocalization of $\mathrm{H}$ into the interstitial space of the bcc cell.

Figure 1 shows the VII"-VII' transition line determined by the AIMD simulations [10]. The latter intersects our 
melting line at $\sim 41 \mathrm{GPa}$, suggesting that ice $\mathrm{VII}^{\prime \prime}$ has a limited domain of stability, ending near $41 \mathrm{GPa}$ and $1400 \mathrm{~K}$. We note that the solid transition temperatures reported in Ref. [20] increase less rapidly with pressure, as depicted by the dash-dotted line in Fig. 1. In such case, the stability domain of ice VII" would extend to higher pressures. Interestingly though, the authors of Ref. [20] ceased to observe the solid transition above $42 \mathrm{GPa}$, and their melting curve presents a change of slope at this pressure, indicative of another triple point.

In conclusion, our experiments have revealed a HT bcc form of ice stable along the melting line above $850 \mathrm{~K}$, transiting to ice VII' at higher pressure through an isostructural, first-order transition. The large entropy increase at the transition, and excellent agreement with AIMD data strongly suggest that the HT phase is the superionic ice VII" phase. The VII'-VII" transition line meets the melting line at $14.6 \mathrm{GPa}$, where a kink is detected in experiment. Ice VII" is observed up to $44 \mathrm{GPa}$ below the melting line, but its stability at higher pressures is questioned. Whether a different phase, such as the predicted fcc solid [52,53], becomes stable and push the melting line to higher temperatures, as suggested by shock experiments $[16,17]$, is an important issue for planetary sciences, and a future challenge for experiments.

We thank F. Ocelli and S. R. Evans for assistance in experiments, and P. Loubeyre for discussions. We acknowledge ESRF and SOLEIL synchrotrons for the provision of beam time (ESRF projects HS-4184, HD-463, and HC-3952, SOLEIL project 99180039), and the Agence Nationale de la Recherche for support under Grants No. ANR 13-BS04-0015 (MOFLEX) and No. ANR 15-CE30-0008-01 (SUPERICES). Computational resources were provided by the Norwegian infrastructure for high-performance computing (NOTUR Grants No. NN9329K and No. NN2916K), and by the CINES supercomputing centre (e Demande d'Attribution de Ressources Informatiques Grant No. A0040810469). J. A. $\mathrm{H}$ acknowledges support from the Research Council of Norway through its Centres of Excellence funding scheme, project No. 223272.

*frederic.datchi@sorbonne-universite.fr

[1] P. Loubeyre, R. LeToullec, E. Wolanin, M. Hanfland, and D. Hausermann, Nature (London) 397, 503 (1999).

[2] W. F. Kuhs, J. L. Finney, C. Vettier, and D. V. Bliss, J. Chem. Phys. 81, 3612 (1984).

[3] J. D. Jorgensen and T. G. Worlton, J. Chem. Phys. 83, 329 (1985).

[4] A. F. Goncharov, N. Goldman, L. E. Fried, J. C. Crowhurst, I. Feng W. Kuo, C. J. Mundy, and J. M. Zaug, Phys. Rev. Lett. 94, 125508 (2005).

[5] E. Sugimura, T. Komabayashi, K. Hirose, N. Sata, Y. Ohishi, and L. S. Dubrovinsky, Phys. Rev. B 82, 134103 (2010).
[6] J.-A. Hernandez and R. Caracas, J. Chem. Phys. 148, 214501 (2018).

[7] W. B. Holzapfel, J. Chem. Phys. 56, 712 (1972).

[8] R. J. Hemley, A. P. Jephcoat, H. K. Mao, C. S. Zha, L. W. Finger, and D. E. Cox, Nature (London) 330, 737 (1987).

[9] E. Wolanin, P. Pruzan, J. C. Chervin, B. Canny, M. Gauthier, D. Häusermann, and M. Hanfland, Phys. Rev. B 56, 5781 (1997).

[10] J.-A. Hernandez and R. Caracas, Phys. Rev. Lett. 117, 135503 (2016).

[11] Y. Takii, K. Koga, and H. Tanaka, J. Chem. Phys. 128, 204501 (2008).

[12] J. L. Aragones and C. Vega, J. Chem. Phys. 130, 244504 (2009).

[13] C. Cavazzoni, G. L. Chiarotti, S. Scandolo, E. Tosatti, M. Bernasconi, and M. Parrinello, Science 283, 44 (1999).

[14] N. Goldman, L. E. Fried, I. Feng W. Kuo, and C. J. Mundy, Phys. Rev. Lett. 94, 217801 (2005).

[15] E. Sugimura, T. Komabayashi, K. Ohta, K. Hirose, Y. Ohishi, and L. S. Dubrovinsky, J. Chem. Phys. 137, 194505 (2012).

[16] M. Millot, S. Hamel, J. R. Rygg, P. M. Celliers, G. W. Collins, F. Coppari, D. E. Fratanduono, R. Jeanloz, D. C. Swift, and J. H. Eggert, Nat. Phys. 14, 297 (2018).

[17] M. Millot, F. Coppari, J. R. Rygg, A. Correa Barrios, S. Hamel, D. C. Swift, and J. H. Eggert, Nature (London) 569, 251 (2019).

[18] J.-F. Lin, E. Gregoryanz, V. V. Struzhkin, M. Somayazulu, H.-K. Mao, and R. J. Hemley, Geophys. Res. Lett. 32, L11306 (2005).

[19] B. Schwager, L. Chudinovskikh, A. G. Gavriliuk, and R. Boehler, J. Phys. Condens. Matter 16, S1177 (2004).

[20] B. Schwager and R. Boehler, High Press. Res. 28, 431 (2008).

[21] F. Datchi, P. Loubeyre, and R. LeToullec, Phys. Rev. B 61, 6535 (2000).

[22] N. Dubrovinskaia and L. Dubrovinsky, High Press. Res. 23, 307 (2003).

[23] M. R. Frank, Y. Fei, and J. Hu, Geochim. Cosmochim. Acta 68, 2781 (2004).

[24] J.-F. Lin, B. Militzer, V. V. Struzhkin, E. Gregoryanz, R. J. Hemley, and H.-K. Mao, J. Chem. Phys. 121, 8423 (2004).

[25] T. Kimura, Y. Kuwayama, and T. Yagi, J. Chem. Phys. 140, 074501 (2014).

[26] M. Ahart, A. Karandikar, S. Gramsch, R. Boehler, and R. J. Hemley, High Press. Res. 34, 327 (2014).

[27] See Supplemental Material at http://link.aps.org/ supplemental/10.1103/PhysRevLett.125.195501 for details on the experimental and theoretical methods, supplemental Tables S1-S2, supplemental Figs. S1-S5, which includes Refs. [28-47].

[28] R. Boehler and K. De Hantsetters, High Press. Res. 24, 391 (2004).

[29] F. Datchi, B. Mallick, A. Salamat, and S. Ninet, Phys. Rev. Lett. 108, 125701 (2012).

[30] J. A. Queyroux, S. Ninet, G. Weck, G. Garbarino, T. Plisson, M. Mezouar, and F. Datchi, Phys. Rev. B 99, 134107 (2019).

[31] K. Takemura and A. Dewaele, Phys. Rev. B 78, 104119 (2008). 
[32] P. Vinet, J. Ferrante, J. R. Smith, and J. H. Rose, J. Phys. C 19, L467 (1986).

[33] L. Benedetti and P. Loubeyre, High Press. Res. 24, 423 (2004).

[34] A. Hammersley, S. O. Svensson, M. Hanfland, A. Fitch, and D. Hausermann, High Press. Res. 14, 235 (1996).

[35] C. Prescher and V. B. Prakapenka, High Press. Res. 35, 223 (2015).

[36] M. Mezouar, P. Faure, W. Crichton, N. Rambert, B. Sitaud, S. Bauchau, and G. Blattmann, Rev. Sci. Instrum. 73, 3570 (2002).

[37] G. Morard, M. Mezouar, S. Bauchau, M. Álvarez-Murga, J.-L. Hodeau, and G. Garbarino, Rev. Sci. Instrum. 82, 023904 (2011).

[38] G. Weck, G. Garbarino, S. Ninet, D. K. Spaulding, F. Datchi, P. Loubeyre, and M. Mezouar, Rev. Sci. Instrum. 84, 063901 (2013).

[39] G. Kresse and J. Furthmüller, Comput. Mater. Sci. 6, 15 (1996).

[40] G. Kresse and J. Furthmüller, Phys. Rev. B 54, 11169 (1996).
[41] P. Hohenberg and W. Kohn, Phys. Rev. 136, B864 (1964).

[42] W. Kohn and L. J. Sham, Phys. Rev. 140, A1133 (1965).

[43] J. P. Perdew, K. Burke, and M. Ernzerhof, Phys. Rev. Lett. 77, 3865 (1996).

[44] M. French and R. Redmer, Phys. Rev. B 91, 014308 (2015).

[45] P. E. Blöchl, Phys. Rev. B 50, 17953 (1994).

[46] G. Kresse and D. Joubert, Phys. Rev. B 59, 1758 (1999).

[47] S. Klotz, K. Komatsu, H. Kagi, K. Kunc, A. SanoFurukawa, S. Machida, and T. Hattori, Phys. Rev. B 95, 174111 (2017).

[48] E. Schwegler, M. Sharma, F. Gygi, and G. Galli, Proc. Natl. Acad. Sci. U.S.A. 105, 14779 (2008).

[49] F. Simon and G. Glatzel, Z. Anorg. Allg. Chem. 178, 309 (1929).

[50] F. Birch, Phys. Rev. 71, 809 (1947).

[51] S. Ninet, F. Datchi, and A. M. Saitta, Phys. Rev. Lett. 108, 165702 (2012).

[52] H. F. Wilson, M. L. Wong, and B. Militzer, Phys. Rev. Lett. 110, 151102 (2013).

[53] M. French, M. P. Desjarlais, and R. Redmer, Phys. Rev. E 93, 022140 (2016). 